\title{
Prescribing errors during hospital inpatient care: factors influencing identification by pharmacists
}

\author{
Mary P. Tully $\cdot$ Iain E. Buchan
}

Received: 11 May 2009/Accepted: 14 September 2009/Published online: 24 September 2009

(C) Springer Science+Business Media B.V. 2009

\begin{abstract}
Objective: To investigate the prevalence of prescribing errors identified by pharmacists in hospital inpatients and the factors influencing error identification rates by pharmacists throughout hospital admission. Setting: 880-bed university teaching hospital in North-west England. Methods: Data about prescribing errors identified by pharmacists (median: 9 (range 4-17) collecting data per day) when conducting routine work were prospectively recorded on 38 randomly selected days over 18 months. Main outcome measures: Proportion of new medication orders in which an error was identified; predictors of error identification rate, adjusted for workload and seniority of pharmacist, day of week, type of ward or stage of patient admission. Results: 33,012 new medication orders were reviewed for 5,199 patients; 3,455 errors (in 10.5\% of orders) were identified for 2,040 patients $(39.2 \%$; median 1 , range $1-12)$. Most were problem orders $(1,456,42.1 \%)$ or potentially significant errors $(1,748,50.6 \%) ; 197(5.7 \%)$ were potentially serious; $1.6 \%(n=54)$ were potentially severe or fatal. Errors were $41 \%$ (CI: 28-56\%) more likely to be identified at patient's admission than at other times, independent of confounders. Workload was the strongest predictor of error identification rates, with $40 \%$ (33-46\%) less errors identified on the busiest days than at other times. Errors identified fell by $1.9 \%(1.5-2.3 \%)$ for every additional chart checked, independent of confounders. Conclusions: Pharmacists routinely identify errors but
\end{abstract}

M. P. Tully $(\bowtie)$

School of Pharmacy and Pharmaceutical Sciences, University of Manchester, Oxford Road, Manchester M13 9PT, UK e-mail: mary.tully@manchester.ac.uk

I. E. Buchan

Northwest Institute for BioHealth Informatics, University of Manchester, Oxford Road, Manchester M13 9PT, UK increasing workload may reduce identification rates. Where resources are limited, they may be better spent on identifying and addressing errors immediately after admission to hospital.

Keywords Hospital admission - Hospital pharmacist . Medicines reconciliation - Prescribing errors .

Workload, United Kingdom

\section{Impact of findings on practice}

- Pharmacists find errors in $10.5 \%$ of new medication orders in hospital. About $7 \%$ are potentially serious or potentially severe.

- In hospital, it is more likely that errors are identified by the pharmacist on admission to hospital than at any other time.

- As workload increases, the identification rate of prescribing errors decreases.

\section{Introduction}

Prescribing errors are common in hospital practice internationally. Errors occurred in $1.5 \%$ of medication orders prescribed during the hospital stay in the United Kingdom (UK) [1] and in up to $6.2 \%$ of medication orders in the United States of America (USA) [2, 3].

Such studies [1-5] have used pharmacists to collect data on errors they identified in the course of routine work. Pharmacists in most UK and US hospitals are responsible for medicines management during admission. Identifying and, more importantly, addressing prescribing errors is central to that role [6]. In 2001, the Audit Commission 
(responsible for ensuring public money in England is spent economically) suggested that pharmacists take a medication history as soon as possible after hospital admission, to ensure accurate transfer of prescribing information between primary and secondary care [7]. This preceded more recent guidance on pharmacists' involvement in medicines reconciliation [8]. Slee et al. demonstrated that pharmacists taking medication histories identify around 1.5 interventions per patient in $38 \%$ of all acute admissions to medical wards [9]. The overall impact this change of focus has had on how pharmacists identify prescribing errors generally is unknown. Nor is it known whether factors such as workload or seniority affect their ability to identify prescribing errors.

\begin{abstract}
Aims
The aims of this study were to investigate the prevalence of prescribing errors identified by pharmacists in hospital inpatients throughout the hospital admission and which factors influenced the error identification rate by pharmacists during their ward visits.
\end{abstract}

\section{Methods}

Using previously described methods [1, 2, 4, 5, 10], pharmacists recorded prescribing errors identified when conducting their routine work on inpatient wards (Table 1). At the study hospital, inpatient medication orders were written by doctors directly onto a combined prescription and nursing administration chart, known as 'the drug chart' (normal UK practice). Pharmacists checked these drug charts on the wards, prior to ordering supplies and signed the drug charts as confirmation. Limited stocks of urgently needed medicines were kept on the wards. Other medicines were dispensed for each individual and labelled with the patient's name. Few drug charts were sent directly to the dispensary by the nursing staff, to avoid their absence when medication administration was necessary. The work of 39 pharmacists was studied on 38 randomly selected days between March 2003 and August 2004 in a 880-bed university teaching hospital. Ethics Committee approval was obtained.

Each weekday was sampled, and a median of 9 pharmacists (range 4-17, depending on factors such as whether it was a public holiday) recorded the prescribing errors they identified during their normal ward visits on that day. All wards visited by pharmacists were included in the study, categorised by speciality. The seniority of the pharmacist was defined as either junior (less than 4 years experience) or senior (4 years experience or more). The pharmacist's "checking workload" per day was defined as the number of patient's drug charts they examined that day. Pharmacists did not have a fixed duration for their ward work; most, however, tried to spend $2-3 \mathrm{~h}$ in the morning and afternoon. After data collection, the data were ordered by the total number of drug charts checked each day and the days were grouped into quartiles.

All types of new orders (i.e. regular, when-required or once-only medication), prescribed on the day of data collection, were clinically checked by the pharmacists, using their routine practice. Intravenously administered drugs and fluids were included, but the latter were rarely checked by the pharmacists. In most, but not all, cases this occurred before medication administration to the patient. For admitted or discharged patients, and when a drug chart was rewritten, 'new medication orders' referred to all medications that were prescribed for the patient at that time. During the patient's stay, they referred to medications newly prescribed on that day. Pharmacists judged whether a prescribing error had occurred and categorised it, using the definition and typology of Dean et al. [1]. Data were collected on the numbers of medication orders checked, whether an error was present and how many, and the nature

Table 1 Standards for provision of pharmacy services at study hospital

\begin{tabular}{|c|c|}
\hline Activity & Standard \\
\hline Taking a medication history & Pharmacists will take and document drug histories of all new patients admitted to their wards \\
\hline $\begin{array}{l}\text { Medication order endorsement by } \\
\text { pharmacist on wards }\end{array}$ & $\begin{array}{l}\text { Pharmacists must amend and sign medication orders to ensure that there is no confusion regarding safe } \\
\text { and effective drug administration }\end{array}$ \\
\hline Monitoring for drug-related problems & $\begin{array}{l}\text { Pharmacists should monitor each patient's drug chart once a day for any drug-related problems were } \\
\text { available and appropriate }\end{array}$ \\
\hline $\begin{array}{l}\text { Ensuring newly prescribed medicines } \\
\text { are safe }\end{array}$ & $\begin{array}{l}\text { Pharmacists should identify patients with newly prescribed medicines and ensure that the medicine is } \\
\text { safe and appropriate for the patient }\end{array}$ \\
\hline Therapeutic drug monitoring & $\begin{array}{l}\text { Pharmacists should follow the detailed guide to therapeutic drug monitoring for drugs with narrow } \\
\text { therapeutic indices }\end{array}$ \\
\hline Pharmaceutical discharge & $\begin{array}{l}\text { Pharmacists should identify patients for discharge and ensure all medicines are accurately prescribed } \\
\text { and patients counselled as appropriate }\end{array}$ \\
\hline
\end{tabular}


and context of the error. Initial and ongoing training in the data collection method was provided via face-to-face meetings and written information, as to what was and was not considered an error [11].

The routine action by a pharmacist on identifying an apparent error, was either to amend the drug chart in green pen (where the prescriber's intention was clear but the medication order was written in a potentially ambiguous way for nursing administration) or to contact the prescriber (where the intention was unclear or appeared to be at fault). Of the latter, only those that both agreed were errors were recorded in this study. The potential severity of the errors was judged from the available data, by two independent pharmacists using the categorization of Lesar and colleagues [2]. Problem orders included, for example, orders that lacked specific dose or dosage strength information or that were written in a way that was unlikely to be carried out, given the nature of the dosage form prescribed. Potentially significant errors included, for example, a dose ordered that was five times or greater than normal, with potential for adverse effects because of the high dose, or the wrong route of administration ordered with potential for increased adverse effects or inadequate therapy. Potentially serious errors included, for example, a dose ordered for a medication with a low therapeutic index that was 4-10 times the normal dose or a route ordered that could potentially produce serious toxic reactions or inadequate therapy for a serious illness. Potentially severe or fatal errors included, for example, a medication ordered that had the potential to produce a severe or life-threatening reaction in the patient. Judgments were made independently, discussed and consensus reached.

The main outcome was the proportion of new medication orders in which a prescribing error was found. Univariate comparisons between categorical variables were made using a chi-square statistic. The multivariate predictors of this proportion were analysed (using Stata version 9.2) under a logistic model using the generalised estimating equation to incorporate observations repeated among patients. Errors were treated as binomial, the link function was logit, and the within-group correlation structure was exchangeable. The results of statistical analyses are reported as the main effect, with a $95 \%$ confidence interval.

\section{Results}

There were 33,012 individual new medication orders reviewed for 5,199 patients (6.3/patient); 3,455 errors (in $10.5 \%$ of orders) were identified for 2,040 patients $(39.2 \%$; median 1, range 1-12). Table 2 shows the commonly identified prescribing errors and Table 3 shows the errors identified at the four different prescribing stages during the patients' hospital admissions, including those identified more commonly at each prescribing stage. Most were classified as problem orders $(1,456,42.1 \%)$ or potentially significant errors $(1,748,50.6 \%)$. Less than $6 \%$ were potentially serious $(197,5.7 \%)$, and $1.6 \%(n=54)$ were potentially severe or fatal.

Table 2 Types of prescribing errors identified, based on work of Dean and colleagues [1]

\begin{tabular}{|c|c|c|}
\hline & Error classification & Total \\
\hline \multicolumn{3}{|c|}{ Need for drug } \\
\hline 1 & Unintentional omission on admission & 525 \\
\hline 2 & Omission from chart at rewrite & 56 \\
\hline 3 & Unintentional omission from discharge prescription & 154 \\
\hline 4 & Premature discontinuation & 22 \\
\hline 5 & Not prescribed when indicated & 136 \\
\hline 6 & Continuation for longer than necessary & 213 \\
\hline 7 & No indication & 74 \\
\hline 8 & Duplication of treatment & 100 \\
\hline Total & & 1,280 \\
\hline \multicolumn{3}{|c|}{ Selection of specific drug } \\
\hline 9 & Significant allergy & 11 \\
\hline 10 & Clinical contra-indication & 24 \\
\hline 11 & Continuing in event of adverse drug reaction & 10 \\
\hline 12 & Contra-indicated due to drug interaction & 18 \\
\hline 13 & Not drug intended & 39 \\
\hline Total & & 102 \\
\hline \multicolumn{3}{|c|}{ Select dosage regimen } \\
\hline 14 & Failure to specify maximum dose & 422 \\
\hline 15 & Failure to take into account drug interaction & 17 \\
\hline 16 & Dose/rate mismatch for infusion & 12 \\
\hline 17 & Ignoring therapeutic drug monitoring level outside range & 24 \\
\hline 18 & Daily dose incorrectly split into doses & 38 \\
\hline 19 & Overdose (not included in 14-18) & 196 \\
\hline 20 & Underdose (not included in 14-18) & 197 \\
\hline Total & & 906 \\
\hline \multicolumn{3}{|c|}{ Administration of drug } \\
\hline 21 & Wrong route & 51 \\
\hline 22 & Wrong formulation & 293 \\
\hline 23 & Administration times incorrect or not specified & 361 \\
\hline 24 & IV instructions incorrect or not specified & 30 \\
\hline 25 & Start date incorrect/missing & 18 \\
\hline Total & & 759 \\
\hline \multicolumn{3}{|c|}{ Provide drug product } \\
\hline 26 & Product or formulation not specified & 100 \\
\hline 27 & Strength or dose not specified (if $>1$ product exists) & 166 \\
\hline 28 & Route unspecified (if $>1$ possible) & 46 \\
\hline 29 & Medication order not signed & 84 \\
\hline 30 & $\mathrm{CD}$ legislation requirements not fulfilled & 14 \\
\hline \multicolumn{2}{|c|}{ Total } & 410 \\
\hline \multicolumn{2}{|c|}{ Overall total } & 3,455 \\
\hline
\end{tabular}


Table 3 Types of prescribing errors identified at different stages of hospital admission

\begin{tabular}{lccccc}
\hline & On admission & During stay & Rewriting chart & Discharge prescription & Total \\
\hline New medication orders checked & 7,139 & 15,387 & 5,017 & 5,480 & 33,012 \\
New medication orders with errors & $1,037(14.5 \%)$ & $1,388(9.0 \%)$ & $370(7.4 \%)$ & $660(12.0 \%)$ & $3,455(10.5 \%)$ \\
Categories of errors & & & & \\
Need for drug & $485(46.8 \%) *$ & $377(27.2 \%)$ & $89(24.1 \%)$ & $329(49.8 \%)^{*}$ & $1,280(37 \%)$ \\
Selection of drug & $19(1.8 \%)$ & $53(3.8 \%)$ & $8(2.2 \%)$ & $22(3.3 \%)$ & $102(3.0 \%)$ \\
Dosage regimen & $245(23.6 \%)$ & $483(34.8 \%)^{*}$ & $80(21.6 \%)$ & $98(14.8 \%)$ & $906(26.2 \%)$ \\
Administration & $183(17.6 \%)$ & $279(20.1 \%)$ & $102(27.6 \%)^{*}$ & $195(29.5 \%)^{*}$ & $759(21.9 \%)$ \\
Provide product & $99(9.5 \%)$ & $204(14.7 \%)$ & $91(24.6 \%)^{*}$ & $16(2.4 \%)$ & $410(11.9 \%)$ \\
\hline
\end{tabular}

* Error categories occurring more commonly in this prescribing stage $(P<0.0001)$

Overall, $44.7 \%$ of patients $(n=2,324)$ had at least one error identified in their prescribed medication at the time of admission. The logistic model showed that the likelihood of errors being identified by the pharmacists shortly after the patient's admission was $41 \%$ (95\% confidence intervals (CI): $28-56 \%$ ) greater than at other times (odds ratio (OR) 1.41, CI 1.28-1.55). This effect was independent of the seniority of the pharmacist, their checking workload, the day of the week and type of ward. The likelihood of senior pharmacists identifying errors was 34\% (22-47\%) greater than junior pharmacists doing so (OR 1.34, CI 1.22-1.47). This was independent of their checking workload, the day of the week, the stage of admission and the type of ward.

The strongest predictor of prescribing error identification rates for the pharmacists was the number of patients whose drug charts were clinically checked. The likelihood of errors being identified was least during the busiest days (i.e. those days categorised in the upper quartile group, based on the total number of drug charts checked that day), with $40 \%$ (33$46 \%)$ less errors identified on those days than at other times (OR 0.60, CI 0.54-0.67). For every additional drug chart checked above a mean number of 27 per pharmacist per day, the likelihood of identifying a prescribing error fell by $1.9 \%$ (1.6-2.3\%) (OR 0.981, CI 0.977-0.984). This was independent of the type of ward, the stage of admission, the day of the week and the seniority of the pharmacist.

Errors were $16 \%$ (5-26\%) less likely to be identified on Fridays than on other days of the week (OR 0.84, CI 0.740.95). This is independent of checking workload, seniority of the pharmacist and stage of admission. There was a significant interaction with the type of ward; the 'Friday effect' was seen on general surgical and care of the elderly wards but not on general medical wards.

\section{Discussion}

This study found that errors were identified by pharmacists in over $10 \%$ of new medication orders and that nearly $40 \%$ of patients admitted to a UK teaching hospital had one or more prescribing errors identified during their stay. There were more errors identified shortly after admission than at any other time during the stay. This affected almost half the patients, but most errors were not potentially serious or severe. Pharmacist seniority and checking workload were strong predictors of error identification rates.

This study has a number of limitations. Data represent the prevalence of prescribing errors that were both identified and recorded by the pharmacists, rather than the prevalence of errors that had occurred in these new medication orders. This is a known limitation with this research method [12]. We used intermittent data collection to reduce fatigue, with ongoing training and feedback to reinforce the need for accurate and complete data recording. It was not possible within the available resources, unfortunately, for another pharmacist to check independently how many errors were not recognised or identified but not recorded. Inclusion of a quality assurance check, to identify missed or unrecorded prescribing errors, has been shown to increase the error identification rate [13]. Thus, the prevalence found is probably an under-estimate of the actual prescribing error rate in hospital inpatients.

As with most previous work [14], this study was conducted in a single teaching hospital, using a single model of pharmacy service delivery, albeit common in the UK. The pharmacists' workload was assessed very crudely, as the number of charts checked per day, and did not include any other work conducted. The data are several years old, potentially limiting their generalisability. In our recent systematic review of the prevalence of prescribing errors from 1985 to 2007, however, there was no noticeable change over time [14]. It could also be argued that the advent of electronic prescribing makes such data obsolete, but this is not commonplace. In the USA, for example, electronic prescribing is available in only $17 \%$ of hospitals and even fewer have a comprehensive electronic patient record [15]. It is likely, therefore, that data about paperbased prescribing errors will still be relevant for some time. 
Severity of identified errors was evaluated by two independent pharmacists; no errors had been judged an error by the data collectors but not an error by these pharmacists. The severity of errors identified in this study was more likely to be either problem orders or potentially significant errors, rather than more serious errors. This is a reflection of the process-based data collection method [12], meaning that errors preventing or delaying medication administration (such as a missing dose or route) were also identified and recorded. This is different from studies that were outcome-based and which only collected data on actual or potential adverse events (such as Bates et al. [16]), which tend to report larger proportions of more severe errors.

A recent systematic review found a median prescribing error rate for hospital inpatients of $7 \%$ (interquartile range: $2-14 \%$ ) of medication orders [14]. The prevalence found in our study was within that range. Such variation may be explained by the different clinical context and data collection methods. Pharmacists working in dispensaries [2, 4, 5, 10], for example, lacking both access to the medical records and face-to-face contact with the patient, may identify fewer errors than do pharmacists working on wards [1]. Our findings are higher than that found by Dean et al. previously in the UK (1.5\%) [1], due to the different data collection methods. Our denominator was the number of new medication orders clinically checked on the day of data collection, corresponding to the number of new orders written in the previous $24 \mathrm{~h}$. For Dean et al. [1], numerator data (errors) were collected by pharmacists, but denominator data (all medication orders written, whether or not seen by a pharmacist) were calculated from a separately collected sample. Data collection fatigue in the pharmacists would thus only affect the numerator data, rather than both the numerator and denominator as in our study.

Pharmacists are the most frequently used healthcare professional to collect data about prescribing errors [14]. Hospital pharmacists in the UK routinely screen in-patient medication orders for errors, prior to requesting supplies to be sent to the ward. Therefore, recording this data is a natural progression of their work. Phansalkar et al. [17] found that pharmacists were the most thorough when conducting chart reviews, compared to other healthcare professionals. Senior, and hence more experienced, pharmacists in this study were more likely to identify errors than were junior pharmacists, suggesting an area where ongoing education should be provided for junior staff.

The pharmacists in our study identified more errors at the time of patients' admissions, regardless of type of ward. In work conducted prior to the Audit Commission recommendation, Dean et al. found more prescribing errors during the hospital stay than at admission [1]. Medication history-taking with all patients at admission was standard practice in this hospital (Table 1) - it was not described as part of their routine pharmacy service by Dean et al. [1]. Slee et al. [9] investigated pharmacist interventions (over $95 \%$ of which appeared due to prescribing errors) solely at the time of admission, when pharmacists conducted medication histories. Their findings were consistent with ours; for example, $38 \%$ of patients required an intervention [9], compared with $44.7 \%$ in our study.

In a systematic review, $27-54 \%$ of patients had at least one error in their medication histories on admission to hospital [18] — our finding of $44.7 \%$ is within this range. It is well recognized that transfer between settings is a common source of medication errors [19, 20]. Sixty percent of patients had an unintended variance [19], and there were two medication errors each time an elderly patient was transferred between primary and secondary care [20]. Thus, the World Health Organization has included the assurance of medication accuracy at transition points in care as one of its 'High 5' patient safety solutions [21].

During this study, pharmacists would obtain and document data about current medication from the patient and/or their general practitioner, check that their drug chart reflected this 'medication history', discuss any discrepancies with the medical team and ensure errors were corrected. This is very similar to medicines reconciliation as defined by the National Prescribing Centre in the UK (collecting information on medication history, checking this list against the current drug chart in the hospital and communicating changes, omissions and discrepancies) [22] and as used in the USA (a process involving verification of use, identification of variances and rectification of errors) [19]. In the USA, the Institute for Healthcare have included medication reconciliation as an initiative to improve patient safety in their ' 5 Million Lives' campaign [23] Its implementation is currently a National Patient Safety Goal of the Joint Commission on Accreditation of Health Care Organizations [24]. The Healthcare Commission, which assesses the performance of healthcare organisations in the UK, has specifically recommended that "weaknesses in transferring information" about medicines should be addressed [25]. The National Institute for Health and Clinical Excellence has hence made recommendations for good practice in this area [8].

The Healthcare Commission suggested that pharmacists made more interventions the longer they spent with each patient [25]. However, the time available to pharmacists is finite. Albeit a crude workload estimate, in this study they identified less errors as the number of drug charts reviewed went up. Barber et al., in the early 1990s, similarly found that there was an inverse relationship between the number of patients that the pharmacist provided a clinical service to and the number of interventions made [26]. In primary care also, community pharmacists' intervention rated decreases with increased dispensing workload [27, 28]. 
Other healthcare professionals have been found to cause more errors when they are working under greater intensity. Workload was an independent risk factor for administration errors on nurses' drug rounds [29]. Junior doctors may make more prescribing errors when they are busiest [30]. Fatigue is recognised as an error-provoking condition for causing both prescribing [31, 32] and medication errors [33]. There is the risk, therefore, that overworked, stressed pharmacists could err by incorrectly identifying errors or missing them completely.

It must be recognised, however, that this study measured only a small part of the pharmacists' workload, namely checking drug charts. This could explain why, on Fridays, independent of workload, fewer errors were identified. It could be hypothesised that there were additional workload expectations on pharmacists at the end of the week, which were not measured in this study. Workload intensity is a factor worthy of further research, to ensure that staff levels are appropriate and the patient-safety role of pharmacists is not compromised.

\section{Conclusions}

Prescribing errors are still commonly identified in UK hospitals, and may be more common than previously thought. Pharmacists are vital in identifying and addressing these errors, but workload may reduce error identification rates. Thus, if resources are limited, they may be better spent on identifying prescribing errors immediately after a patient is admitted to hospital than at any other time.

Acknowledgments We thank all the pharmacists that participated in the study and contributed to the data collection.

Funding Data collection funded internally; data analysis funded by Patient Safety Research Programme (as part of grant PS020).

Conflicts of interest statement None.

\section{References}

1. Dean B, Schachter M, Vincent C, Barber N. Prescribing errors in hospital inpatients: their incidence and clinical significance. Qual Saf Health Care. 2002;11(4):340-4.

2. Lesar TS, Lomaestro BM, Pohl H. Medication-prescribing errors in a teaching hospital. A 9-year experience. Arch Intern Med. 1997; 157:1569-76.

3. Bobb A, Gleason K, Husch M, Feinglass J, Yarnold PR, Noskin GA. The epidemiology of prescribing errors: the potential impact of computerized prescriber order entry. Arch Intern Med. 2004;164(7):785-92.

4. Blum KV, Abel SR, Urbanski CJ. Medication error prevention by pharmacists. Am J Hosp Pharm. 1988;45:1902-3.
5. Folli HL, Poole RL, Benitz WE. Medication error prevention by clinical pharmacists in two children's hospitals. Pediatrics. 1987;79:718-22.

6. Hawkey CJ, Hodgson S, Norman A, Danseshmend TK, Garner ST. Effect of reactive pharmacy intervention on quality of hospital prescribing. BMJ. 1990;300:986-90.

7. Audit Commission. A spoonful of sugar-improving medicines management in hospitals. London: Audit Commission; 2001.

8. National Institute for Health and Clinical Excellence, National Patient Safety Agency. Technical patient safety solutions for medicines reconciliation on admission of adults to hospital. London: National Institute for Health and Clinical Excellence; 2007.

9. Slee A, Farrar K, Hughes D, Constable S. Optimising medical treatment-how pharmacist-acquired medication histories have a positive impact on patient care. Pharm J. 2006;277:737-9.

10. Lesar TS, Briceland L, Delcoure K, Parmalee JC, Masta-Gornick $\mathrm{V}$, Pohl $\mathrm{H}$. Medication-prescribing errors in a teaching hospital. JAMA. 1990;263:2329-34.

11. Dean B, Barber N, Schachter M. What is a prescribing error? Qual Health Care. 2000;9:232-7.

12. Dean Franklin B, Vincent C, Schachter M, Barber N. The incidence of prescribing errors in hospital inpatients: an overview of the research methods. Drug Saf. 2005;28(10):891-900.

13. Donyai P, O'Grady K, Jacklin A, Barber N, Franklin BD. The effects of electronic prescribing on the quality of prescribing. $\mathrm{Br} \mathrm{J}$ Clin Pharmacol. 2008;65(2):230-7.

14. Lewis P, Dornan T, Taylor D, Tully MP, Wass V, Ashcroft DM. Systematic review of the prevalence, incidence and nature of prescribing errors in hospital inpatients. Drug Saf. 2009;32: 379-89.

15. Jha AK, DesRoches CM, Campbell EG, Donelan K, Rao SR, Ferris TG, et al. Use of electronic health records in U.S. hospitals. N Engl J Med. 2009;360(16):1628-38.

16. Bates DW, Cullen DJ, Laird N, Petersen LA, Small SD, Servi D, et al. Incidence of adverse drug events and potential adverse drug events. JAMA. 1995;274:29-34.

17. Phansalkar S, Hoffman JM, Nebeker JR, Hurdle JF. Pharmacists versus nonpharmacists in adverse drug event detection: a metaanalysis and systematic review. Am J Health Syst Pharm. 2007;64(8):842-9.

18. Tam VC, Knowles SR, Cornish PL, Fine N, Marchesano R, Etchells EE. Frequency, type and clinical importance of medication history errors at admission to hospital: a systematic review. CMAJ. 2005;173(5):510-5.

19. Vira T, Colquhoun M, Etchells E. Reconcilable differences: correcting medication errors at hospital admission and discharge. Qual Saf Health Care. 2006;15(2):122-6.

20. Midlov P, Bergkvist A, Bondesson A, Eriksson T, Hoglund P. Medication errors when transferring elderly patients between primary health care and hospital care. Pharm World Sci. 2005;27:116-20.

21. World Health Organisation. Action on patient safety-high $5 \mathrm{~s}$. World alliance for patient safety. 2009. URL: http://www. who.int/patientsafety/solutions/high5s/en/index.html. Accessed 8 Jul 2009.

22. National Prescribing Centre. Medicines reconciliation: a guide to implementation. Liverpool: National Prescribing Centre; 2007.

23. Institute for Healthcare Improvement. Protecting 5 million lives from harm. Institute for Healthcare Improvement. 2009. URL: http://www.ihi.org/IHI/Programs/Campaign/. Accessed 8 Jul 2009.

24. Joint Commission on Accreditation of Healthcare Organizations. Hospitals' national patient safety goals. Joint Commission on Accreditation of Healthcare Organizations. 2009. URL: 
http://www.jointcommission.org/PatientSafety/

NationalPatientSafetyGoals/ Accessed 8 Jul 2009.

25. Healthcare Commission. The best medicine. The management of medicines in acute and specialist trusts. London: Commission for Healthcare Audit and Inspection; 2007.

26. Barber N, Blackett A, Batty R. Does a high workload decrease ward pharmacists' clinical monitoring? Int J Pharm Pract. 1993;2(3):152-5.

27. Rupp MT, DeYoung M, Schondelmeyer SW. Prescribing problems and pharmacist interventions in community practice. Med Care. 1992;30:926-40.

28. Hawksworth GM, Corlett AJ, Wright DJ, Chrystyn H. Clinical pharmacy interventions by community pharmacists during the dispensing process. Br J Clin Pharmacol. 1999;47:695-700.

29. Tissot E, Cornette C, Limat S, Mourand JL, Becker M, Etievent $\mathrm{JP}$, et al. Observational study of potential risk factors of medication administration errors. Pharm World Sci. 2003; 25(6):264-8.

30. Davydov L, Caliendo G, Mehl B, Smith LG. Investigation of correlation between house-staff work hours and prescribing errors. Am J Health Syst Pharm. 2004;61(11):1130-4.

31. Coombes ID, Stowasser DA, Coombes JA, Mitchell C. Why do interns make prescribing errors? A qualitative study. Med J Aust. 2008;188(2):89-94.

32. Dean B, Schachter M, Vincent C, Barber N. Causes of prescribing errors in hospital inpatients-a prospective study. Lancet. 2002;359:1373-8.

33. Nichols P, Copeland TS, Craib IA, Hopkins P, Bruce DG. Learning from error: identifying contributory causes of medication errors in an Australian hospital. Med J Aust. 2008; 188(5):276-9. 